\title{
Absorption chez l'homme du caséinate de sodium et des protéines ultrafiltrées de lait
}

\author{
par \\ M. J. BEAUFRAND, B. POULLAIN, H. M. BAU \\ et G. DEBRY
}

Les modifications post-prandiales des concentrations des acides aminés circulants, ont fait l'objet d'études établissant leur corrélation avec l'aminogramme et la digestibilité de la protéine ingérée, ainsi que le taux protéique de la ration.

Depuis que Frame (1958), Gupta (1958) et Longenecker (1959) ont établi cette relation, de nombreux travaux ont repris ce modèle (Harper, 1968 ; Adibi, 1973 ; Weller, 1973).

Cependant la présence de sécrétions azotées endogènes (enzymes, desquamation des muqueuses), dont l'importance est controversée par les auteurs (Nixon, 1970 ; Nasset, 1972 ; Johansson, 1975), les différences dans la vitesse d'absorption des acides aminés dues aux inter-relations entre les nutriments (Rossiter, 1974), le rôle de la barrière hépatique, expliquent les disparités entre le profil des acides aminés circulants et l'aminogramme de la protéine étudiée (Weller, 1973). L'aminoacidémie post-prandiale a été utilisée pour déterminer le besoin en certains acides aminés (Young, 1973) et l'acide aminé limitant. Graham (1973), constate chez l'enfant, qu'après consommation d'un repas contenant une protéine déficiente en acides aminés soufrés, la concentration plasmatique diminue au-dessous du niveau des témoins 3 à 4 h après le repas. Vaughan (1976) à partir de l'aminoacidémie post-prandiale consécutive à l'ingestion de $50 \mathrm{~g}$ de lactalbumine met en évidence les altérations de la disponibilité des acides aminés provoquées par le chauffage.

\footnotetext{
Travail du Département de Nutrition et des Maladies métaboliques de l'Université de Nancy I et du Groupe de Recherche en Nutrition et Diététique de I'I.N.S.E.R.M. U 59. Directeur : professeur G. Debry, 40, rue Lionnois - 54000 Nancy.

Travail réalisé dans le cadre du contrat D.G.R.S.T. $\mathrm{n}^{\circ}$ 74.7.1047.
} 
L'aminoacidémie post-prandiale a également été utilisée pour comparer la vitesse de digestion de différents types de protéines.

La rapidité d'assimilation présente un intérêt particulier pour des produits diététiques destinés à des sujets présentant des troubles du transit. Silk (1975) a mis en évidence à l'aide des variations de l'aminoacidémie totale, l'absorption plus rapide d'un hydrolysat de caséine par rapport au mélange des acides aminés constitutifs de la caséine. Il attribue la différence constatée à la présence d'oligopeptides qui sont absorbés de façon compétitive avec les acides aminés. A notre sens, une telle approche est la seule qui permette de différencier chez l'homme, les protéines en fonction de leur vitesse de digestion, vitesse liée à l'hydrolyse et à l'absorption.

En résumé, la signification nutritionnelle des variations de l'aminoacidémie post-prandiale peut être envisagée d'une part pour la détermination de l'acide aminé limitant d'une protéine, d'autre part pour différencier la vitesse d'absorption des protéines.

\section{MATERIEL ET METHODES}

\subsection{Technologie des produits}

- protéines de lait ultrafiltrées* : l'ultrafiltration fondée sur le principe de l'osmose inverse utilise des membranes sous pression ; les substances de bas poids moléculaire du lait (lactose, sels minéraux...) sont séparées de la phase contenant les protéines ;

- nous avons pris comme témoin le caséinate de sodium obtenu par précipitation des protéines de lait en milieu acide.

\subsection{Protocole}

Le petit-déjeuner de la première épreuve est composé uniquement de $22 \mathrm{~g}$ de caséinate de sodium, apportant $20 \mathrm{~g}$ de protéines.

Le petit-déjeuner de la deuxième épreuve est composé uniquement de $26 \mathrm{~g}$ de protéines de lait ultrafiltrées, apportant $20 \mathrm{~g}$ de protéines, $3 \mathrm{~g}$ de sucres.

Afin d'assurer avant chaque épreuve la constance et l'égalité des apports alimentaires en protéines, il est réalisé $2 \mathrm{j}$ d'alimentation contrôlée dont la composition est précisée dans le tableau $1 . \mathrm{La}$ veille de l'épreuve, le repas du soir apporte essentiellement des glucides et une quantité minimum de protéines, afin d'assurer un taux sanguin basal minimum d'acides aminés avant l'épreuve et d'éviter un catabolisme tissulaire.

\footnotetext{
* Fournies par le laboratoire de technologie laitière de l'I.N.R.A.
} 
TABLEAU 1. - Séquences diététiques

\begin{tabular}{|c|c|c|c|}
\hline Premier jour & Protides g. & Lipides $\mathrm{g}$. & Glucides g. \\
\hline Petit déjeuner & 20 & 16 & 65 \\
\hline Repas de midi & 39 & 44 & 128 \\
\hline Repas du soir & 41 & 47 & 125 \\
\hline Total & 100 & 107 & 318 \\
\hline Niveau calorique & 400 & 963 & 1272 \\
\hline Deuxième jour & Protides g. & Lipides g. & Glucides g. \\
\hline Petit déjeuner & 20 & 16 & 65 \\
\hline Repas de midi & 38 & 47 & 134 \\
\hline Repas du soir & 9 & 44 & 150 \\
\hline Total & 67 & 107 & 349 \\
\hline Niveau calorique & 268 & 963 & 1396 \\
\hline
\end{tabular}

Des prélèvements de sang veineux sont effectués aux temps suivants : à jeun, $60 \mathrm{mn}, 90 \mathrm{mn}$ et $120 \mathrm{mn}$ après l'ingestion des protéines.

\subsection{Dosages}

\subsubsection{Dosage des acides aminés sanguins}

Les $5 \mathrm{ml}$ de sang recueillis sur EDTA (0,2 g/tube) sont centrifugés, et le sérum est traité à l'acide sulfosalicylique $(100 \mathrm{mg} / \mathrm{ml})$, pour déféquer les protéines plasmatiques. $100 \mu \mathrm{l}$ de plasma déféqué sont déposés sur l'auto-analyseur d'acides aminés Technicon TSM.

Afin d'expliquer les résultats obtenus, nous avons étudié les caractéristiques de solubilité et de digestion enzymatique des produits. 


\subsubsection{Solubilité}

Les poudres de caséinate de sodium et de protéines de lait ultrafiltrées sont mises en contact avec une solution aqueuse d'HCL à $\mathrm{pH} 4(\mathrm{pH}$ gastrique en début de digestion). Après différents temps de contact, les solutions sont filtrées et les protéines insolubles sont séchées et pesées.

\subsubsection{Digestions enzymatiques}

Chaque protéine (caséinate de sodium et protéines ultrafiltrées de lait) à la concentration de $20 \mathrm{mg}$ de substrat $/ \mathrm{ml}$ est mise en solution à $37^{\circ} \mathrm{C}$ :

- en milieu tampon Tris $(0,05 \mathrm{M}-\mathrm{pH} 8,2)$ en présence de trypsine (SERVA Réf. : 37260$)$;

- en milieu $\mathrm{HCl}(0,06 \mathrm{~N}$ - pH 1,8$)$ en présence de pepsine (SERVA Réf. : 31 820).

Le rapport enzyme/substrat est égal à 2,5/1000 pour la trypsine et $2 / 1000$ pour la pepsine.

On laisse agir l'enzyme $10 \mathrm{mn}, 20 \mathrm{mn}, 30 \mathrm{mn}, 60 \mathrm{mn}$ et $240 \mathrm{mn}$, puis la réaction est stoppée par addition d'acide trichloroacétique (12 p. 100).

L'azote non protéique est dosé par micro-Kjeldahl.

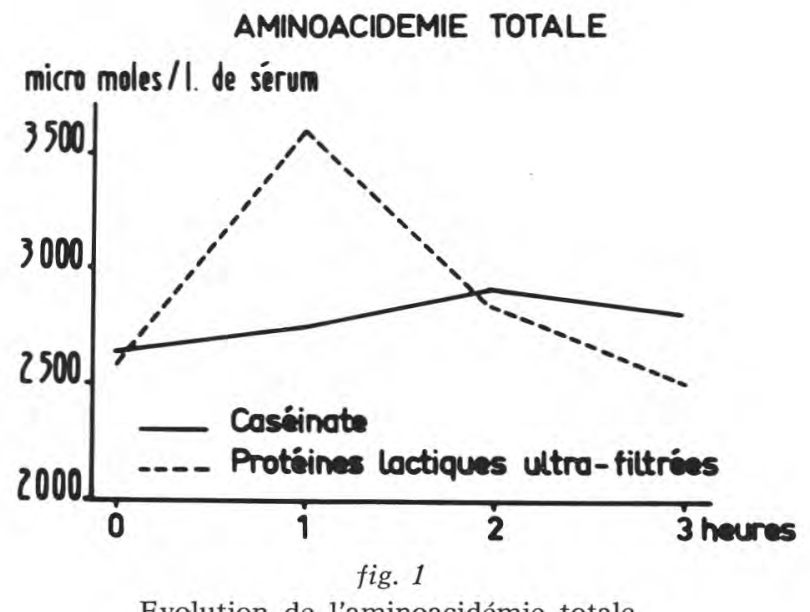

Evolution de l'aminoacidémie totale

\section{RESULTATS}

Lors de la première épreuve, l'aminoacidémie augmente $1 \mathrm{~h}$ après l'ingestion du caséinate de sodium, et atteint son maximum après $2 \mathrm{~h}$.

Après l'ingestion des protéines ultrafiltrées, l'augmentation de l'aminoacidémie est maximum $1 \mathrm{~h}$ après leur ingestion (fig. 1, tab. 1). Lors des deux épreuves, l'évolution des acides aminés indispensables 
et des acides aminés non indispensables est parallèle. L'évolution des différents acides aminés est homogène et ne présente pas de variations individuelles notables (tab. 2).

La détermination de la solubilité du caséinate de sodium et des protéines ultrafiltrées à pH 4 montre que 99 p. 100 des protéines ultrafiltrées sont solubilisées après $15 \mathrm{mn}$, alors que ce pourcentage n'est que de 77 p. 100 avec le caséinate de sodium (tab. 3).

Après $1 \mathrm{~h}$ d'agitation, les protéines ultrafiltrées sont entièrement solubilisées, ainsi que 92 p. 100 du caséinate de sodium.

Sur les courbes de libération d'azote non protéique, au cours des digestions enzymatiques, on observe que la digestion trypsique des protéines ultrafiltrées de lait est plus faible que celle du caséinate de sodium. Après $2 \mathrm{~h}$ de digestion trypsique, seulement 32,5 p. 100 des protéines ultrafiltrées sont digérées contre 53 p. 100 pour le caséinate de sodium (fig. 2).

\section{DISCUSSION}

La phase essentielle de la digestion des protéines chez l'homme est intestinale (Adibi, 1976). Bien que le processus global de digestion (hydrolyse et absorption) soit rapide, et atteigne son maximum $45 \mathrm{mn}$ après l'ingestion (Crane, 1960), le facteur primordial de la régulation de l'absorption chez l'homme est la vitesse de vidange gastrique (Johansson, 1975), ainsi que la compétition entre les mono-saccharides

\section{DIGESTIONS ENZYMATIQUES}

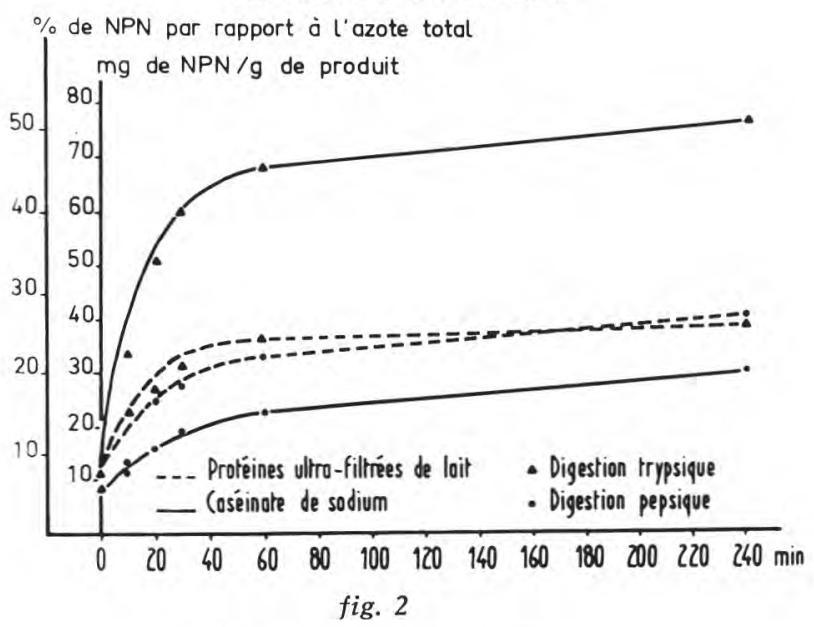

Digestions enzymatiques 
TABLEAU 2. - Acides aminés sanguins $\mu \mathrm{M} / \mathrm{ml}$

\begin{tabular}{|c|c|c|c|c|c|c|c|c|c|c|c|c|c|c|c|c|}
\hline & \multicolumn{8}{|c|}{ I. Caséinate de sodium } & \multicolumn{8}{|c|}{ II. Protéines de lait ultrafiltrées } \\
\hline & $A j$ & eun & $\mathrm{T}=$ & $1 \mathrm{~h}$ & $\mathrm{~T}=$ & $2 \mathrm{~h}$ & $\mathrm{~T}=$ & $3 \mathrm{~h}$ & $A j$ & eun & $\mathrm{T}=$ & $1 \mathrm{~h}$ & $\mathrm{~T}=$ & $2 \mathrm{~h}$ & $\mathrm{~T}=$ & $3 \mathrm{~h}$ \\
\hline & Moy. & SEM & Moy. & SEM & Moy. & SEM & Moy. & SEM & Moy. & SEM & Moy. & SEM & Moy. & SEM & Moy. & SEM \\
\hline $\begin{array}{l}\text { Lysine } \\
\text { Histidine } \\
\text { Arginine } \\
\text { Asparagine } \\
\text { Sérine } \\
\text { Thréonine } \\
\text { Glycine } \\
\text { Alanine } \\
\text { Valine } \\
\text { Méthionine } \\
\text { Iso-leucine } \\
\text { Tyrosine } \\
\text { Leucine } \\
\text { Phényl-alanine } \\
\text { Acides aminés indispensables } \\
\text { Acides aminés totaux }\end{array}$ & $\begin{array}{r}234 \\
117 \\
130 \\
443 \\
207 \\
125 \\
187 \\
381 \\
280 \\
25 \\
72 \\
77 \\
132 \\
60 \\
1127 \\
2470\end{array}$ & $\begin{array}{r}44 \\
8 \\
12 \\
28 \\
6 \\
13 \\
5 \\
13 \\
14 \\
0,1 \\
3 \\
2 \\
8 \\
3 \\
89 \\
58\end{array}$ & $\begin{array}{r}239 \\
113 \\
117 \\
478 \\
236 \\
158 \\
212 \\
521 \\
238 \\
24 \\
81 \\
92 \\
140 \\
86 \\
1130 \\
2735\end{array}$ & $\begin{array}{r}60 \\
4 \\
20 \\
120 \\
45 \\
25 \\
31 \\
26 \\
45 \\
5 \\
12 \\
28 \\
21 \\
4 \\
192 \\
212\end{array}$ & $\begin{array}{r}294 \\
137 \\
138 \\
462 \\
209 \\
137 \\
239 \\
525 \\
279 \\
34 \\
103 \\
102 \\
170 \\
68 \\
1325 \\
2897\end{array}$ & $\begin{array}{r}35 \\
9 \\
40 \\
88 \\
34 \\
26 \\
55 \\
84 \\
51 \\
7 \\
11 \\
24 \\
22 \\
10 \\
180 \\
373\end{array}$ & $\begin{array}{r}272 \\
133 \\
122 \\
490 \\
228 \\
166 \\
201 \\
411 \\
246 \\
35 \\
98 \\
88 \\
160 \\
60 \\
1214 \\
2710\end{array}$ & $\begin{array}{r}30 \\
12 \\
30 \\
100 \\
50 \\
24 \\
28 \\
31 \\
24 \\
9 \\
4 \\
14 \\
9 \\
5 \\
95 \\
211\end{array}$ & $\begin{array}{r}245 \\
110 \\
117 \\
460 \\
178 \\
121 \\
230 \\
520 \\
211 \\
29 \\
77 \\
93 \\
137 \\
72 \\
1091 \\
2600\end{array}$ & $\begin{array}{r}30 \\
6 \\
19 \\
38 \\
17 \\
42 \\
38 \\
63 \\
30 \\
6 \\
6 \\
10 \\
15 \\
19 \\
59 \\
150\end{array}$ & $\begin{array}{r}424 \\
126 \\
150 \\
646 \\
307 \\
163 \\
205 \\
540 \\
374 \\
39 \\
159 \\
102 \\
268 \\
69 \\
1711 \\
3572\end{array}$ & $\begin{array}{r}50 \\
3 \\
24 \\
72 \\
70 \\
10 \\
24 \\
25 \\
56 \\
15 \\
13 \\
19 \\
21 \\
5 \\
155 \\
381\end{array}$ & $\begin{array}{r}356 \\
173 \\
124 \\
513 \\
278 \\
148 \\
150 \\
397 \\
244 \\
26 \\
106 \\
79 \\
193 \\
58 \\
1359 \\
2845\end{array}$ & $\begin{array}{r}30 \\
57 \\
8 \\
74 \\
54 \\
26 \\
19 \\
21 \\
4 \\
10 \\
6 \\
16 \\
15 \\
6 \\
60 \\
182\end{array}$ & $\begin{array}{c}238 \\
127 \\
119 \\
411 \\
226 \\
142 \\
168 \\
417 \\
227 \\
21,5 \\
95 \\
70 \\
174 \\
47 \\
1118,5 \\
2482\end{array}$ & $\begin{array}{c}25 \\
29 \\
7 \\
96 \\
47 \\
17 \\
19 \\
5 \\
15 \\
0,8 \\
9 \\
15 \\
19 \\
7 \\
100 \\
172\end{array}$ \\
\hline
\end{tabular}




\section{TABLEAU 3}

Solubilité des protéines à $\mathrm{pH} 4$

\begin{tabular}{l|c|c}
\hline & Protéines ultrafiltrées de lait & Caséinate de sodium \\
\hline & & \\
$15 \mathrm{mn}$ & 99 p. 100 & 77 p. 100 \\
$30 \mathrm{mn}$ & 99,7 p. 100 & 85 p. 100 \\
$60 \mathrm{mn}$ & 99,8 p. 100 & 92 p. 100 \\
& & \\
\hline
\end{tabular}

\section{TABLEAU 4}

Composition des protéines en acides aminés

\begin{tabular}{l|c|c}
\hline & $\begin{array}{c}\text { Protéines ultrafiltrées } \\
\text { p. 100 d'acides aminés pour } \\
100 \mathrm{~g} \text { de protéines }\end{array}$ & $\begin{array}{c}\text { Caséinate de sodium } \\
\text { p. 100 d'acides aminés pour } \\
100 \mathrm{~g} \text { de protéines }\end{array}$ \\
\cline { 2 - 3 } & & \\
Lysine & 10,3 & \\
Histidine & 2,7 & 8,5 \\
Arginine & 3,1 & 3,6 \\
Acide aspartique & 11,3 & 3,9 \\
Thréonine & 5,3 & 6,8 \\
Sérine & 6,8 & 3,8 \\
Acide glutamique & 18,2 & 7,0 \\
Glycine & 2,5 & 16,3 \\
Alanine & 5,3 & 2,2 \\
Valine & 6,4 & 3,1 \\
Iso-leucine & 6,6 & 6,9 \\
Leucine & 10,5 & 5,6 \\
Tyrosine & 3,7 & 9,3 \\
Phényl-alanine & 3,9 & 7,3 \\
& & 6,4 \\
\hline
\end{tabular}

et les acides aminés lors de la phase de transfert actif à travers les cellules de la muqueuse intestinale (Rossiter, 1974). Ces différents points ont été synthétisés par Fauconneau (1970).

Dans notre étude, l'influence sur la vidange gastrique des nutriments associés aux protéines ultrafiltrées (traces de lactose, matières grasses), peut être négligée compte tenu de leur faible concentration. 
L'augmentation maximum de l'aminoacidémie se produit $1 \mathrm{~h}$ après l'ingestion pour les protéines ultrafiltrées, et $2 \mathrm{~h}$ après pour le caséinate de sodium. Ce résultat est en contradiction avec ceux des digestions enzymatiques in vitro. Paradoxalement, les protéines ultrafiltrées qui sont digérées rapidement chez l'homme, ont une vitesse de digestion trysique in vitro très lente. En fait, la phase de digestion gastrique étant négligeable devant la phase intestinale (Crane, 1960), cette différence de digestibilité constatée in vitro ne semble pas présenter de signification physiologique. En outre, la vitesse de vidange gastrique qui conditionne la vitesse d'absorption des protéines, est elle-même fonction de la solubilité des aliments se trouvant dans l'estomac. La solubilité du caséinate de sodium étant inférieure à celle des protéines ultrafiltrées, on peut penser que ce facteur conditionne les différences entre ces deux protéines.

Bien que la vitesse d'hydrolyse de ces deux protéines ne doivent pas être intrinséquement différente compte tenu de la meilleure digestibilité du caséinate constaté in vitro, le temps d'absorption des protéines ultrafiltrées est nettement plus court.

Les caractéristiques de digestibilité des protéines de lait ultrafiltrées font de ce produit une matière première intéressante dans des produits diététiques destinés à des sujets présentant des troubles du transit.

\section{S u $\mathrm{m} \mathbf{m}$ a r y}

\section{ABSORPTION OF SODIUM CASEINATE AND ULTRAFILTRED MILK PROTEINS IN MAN}

The absorption speed of ultrafiltred milk proteins is compared with that of sodium caseinate by measurement of post-prandial aminoacidemia. Contrary to the results of in vitro pepsic and trypsic digestion the ultrafiltred milk proteins are more quickly digested.

This result is discussed with regard to the two proteins solubility.

Reçu pour publication en mai 1977.

\section{Bibliographie}

[1] Adibi (S. A.) and Mercer (D. W.) (1973). - Protein digestion in human intestine as reflected in luminal, mucosal and plasma amino acid concentrations after meals. J. Clin. Invest., 52, 1586-1794.

[2] Adibi (S. A.) (1976). - Intestinal phase of protein assimilation in man. Am. J. Clin. Nutr., 29, 205-215.

[3] Crane (C. W.) and Neuberger (A.) (1960). - The digestion and absorption of protein by normal man. Biochem. J., 74, 313-323. 
[4] Fauconneau (G.) and Michel (M. C.) (1970). - The role of gastrointestinal tract in the regulation of protein metabolism. In " Mammalian protein Metabolism ", H. N. Munro, Ed. Academic Press, New York, IV, 481-522.

[5] Frame (E.) (1958). - The levels of individual free amino acids in the plasma of normal man at various intervals after a high protein meal. J. Clin. Invest., 37, 1710-1723.

[6] Graham (G. C.) and Placko (R.P.) (1973). - Post-prandial plasma free methionine as an indicator of dietary methionine adequacy in the human infant. J. Nutr., 103, 1347-1351.

[7] Gupta (J. D.), Dakroury (A. M.) and HaRPer (A. E.) (1958). - Observation on protein digestion in vivo : I. Rate of disappearance of ingested protein from the gastrointestinal tract. J. Nutr., 64, 447-456.

[8] Harper (A. E.) (1968). - Diet and plasma amino acids. Am. J. of Cl. Nutr., $21,5,358-366$.

[9] Johansson (C.) (1975). - Characteristics of the absorption pattern of sugar, fat, and protein from composite meals in man. A quantitative study. Scand. J. of Gastroent., 10, 33-42.

[10] Longenecker (J. B.) and Hause (N. L.) (1959). - Relationship between plasma amino acids and composition of the ingested protein. Arch. Biochem., Biophys., 84, 46-59.

[11] NASset (E. S.) (1972). - Amino acid homeostasis in the gut lumen and its nutritional significance. Wld. Rev. Nutr. Diet., 14, 134-153.

[12] Nixon (S. E.) and MAwLr (G. E.) (1970). - The digestion and absorption of protein in man. 1. The site of absorption. 2. The form in which digested protein is absorbed. Brit J. Nutr., 24, 227-240, 241-258.

[13] Rossiter (M. A.), Palmer (T.), Evans (K.) and Whartow (B. A.) (1974). - The short term response to a drink of milk, lactose or casein in children with apparently normal gastrointestinal tracts. Br. J. Nutr., 32, 605-613.

[14] SILK (D. B. A.) and CLARK (M. L.) (1975). - Jejunal absorption of an amino acid mixture simulating casein and an enzymatic hydrolysate of casein prepared for oral administration to normal adults. Br. J. Nutr., 33, 95-100.

[15] Vaughan (D. A.), Womack (M.) and Mc Clain (P. E.) (1974). - Plasma free amino acid levels in human subjects after consuming meals containing lactalbumin, baked lactalbumin or no protein. Fed. Proc., 33, 712.

[16] Weller (C. A.), Margen (S.), Calloway (D. H.) and Meissner (E. F.) (1973). Serum amino acids in young men consuming diet, differing in level and pattern of amino acids. Am. J. Cl. Nutr., 26, 722-727.

[17] Young (V. R.), Tontisirin (K.), Ozalp (I.), Lakshmanan (F.) and Scrimshaw (N.S.) (1973). - Plasma amino acid response curve and amino acid requirements in young men: valine and lysine. J. Nutr., 102, 1159-1170. 